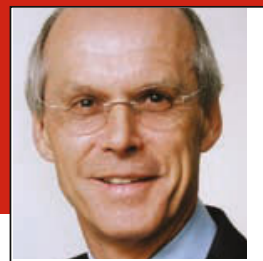

Prof. Dr. med. H. S. FüeßI

Isar-AmperKlinikum, KI. MünchenOst, Haar

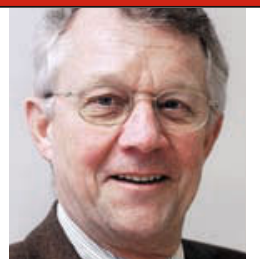

Prof. Dr. med. H. Holzgreve Internist, Kardiologische Praxis, München

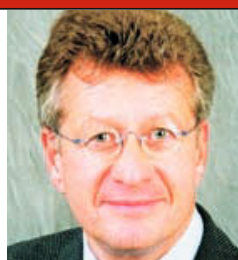

Dr. med. P. Stiefelhagen

Westerwald

Krankenhaus, Hachenburg

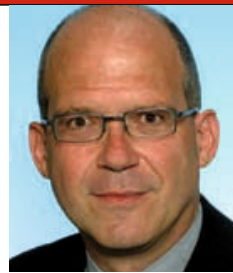

Dr. med. J. Zeeh

Geriatrische

Fachklinik

Georgenhaus, Meiningen

\title{
Ein Statin zur fettreichen Mahlzeit: eine Provokation?
}

Die Empfehlung englischer Kardiologen, das Risiko ungesunder Mahlzeiten durch die gleichzeitige Einnahme eines Statins zu neutralisieren, wird unter Kollegen nicht viele Freunde finden.

— Ferenczi et al. haben einer Studie an 43757 gesunden Probenden entnommen, dass der Konsum von 89 statt $53 \mathrm{~g}$ Fett bzw. 4,3 statt 1,5 g Transfettsäuren täglich (jeweils oberste bzw. unterste Quintile) das Risiko für ein tödliches oder nicht tödliches koronares Ereignis um 23 bzw. 40\% erhöht.

Wer einen McDonald Viertelpfünder (Hamburger Royal TS) mit Käse und Milchshake (Fettgehalt $36 \mathrm{~g}$ ) verzehrt, gelangt somit von der untersten in die oberste Quintile. Andererseits leiten die Autoren aus den großen Interventionsstudien mit Lipidsenkern ab, dass eine Tablette Statin den Schaden eines Hamburgers ziemlich genau neutralisiert.

\section{Kommentar}

Die Autoren, deren Berechnungen nicht ohne weiteres verständlich sind, diskutieren mögliche Einwände. So betrachten sie ungesunde Ernährung als die Summe ungesunder Einzelmahlzeiten. Wer nur einmal im Jahr einen Hamburger isst, hat ein geringes Risiko und verbessert

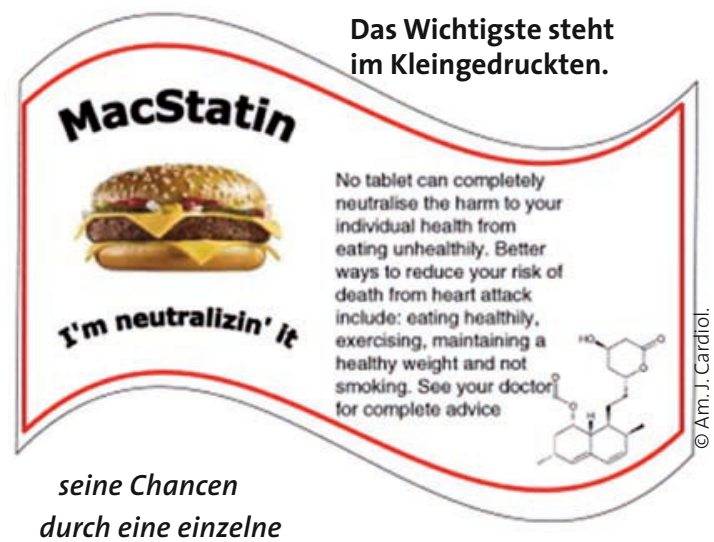

Diabetes und kardiovaskuläre Erkrankungen epidemisch sind. Die Autoren wollen die Menschen, die für Verhaltsänderungen weder die Kraft noch den Willen aufbringen, nicht im Stich lassen. Sie verweisen auf Raucher sowie Motorrad- und Autofahrer, die ebenfalls trotz aller Warnungen ein Risiko eingehen. Ihnen werden Maßnahmen zur Risikominderung angeboten oder sogar gesetzlich oktroyiert. Airbag, Helm und SicherStatindosis nicht. Je häufiger jemand in einer Fast-Food-Kette einkehrt, um so stärker erhöht er sein Risiko und um so sinnvoller wird die gleichzeitige Statineinnahme. Zwischen regelmäßiger Einnahme bei dauerhaft ungesunder Lebensweise und gelegentlicher Einnahme bei mehr oder weniger häufigen Diätfehlern sehen sie keinen prinzipiellen Unterschied. Schließlich wird die Mortalität nach Myokardinfarkt auch durch unregelmäßige Statineinnahme - wenn auch geringer-gesenkt.

Vernünftige Lebensweise wird jedem dringend empfohlen und ungesundes Verhalten kann nicht durch Medikamente kompensiert werden. Das bekräftigen auch die Autoren ausdrücklich. Doch solche Appelle fruchten wenig: Denn sehr viele Menschen leben ungesund, sodass Übergewicht, heitsgurt entsprechen dem Statin beim Diätfehler.

Die Autoren haben auch schon eine Idee stehen viele kostenlose, teils ungesunde Zutaten wie Ketchup, Gewürze, Mayonnaise und Salz auf dem Tisch. Da könnte genauso gut das Statinpulver stehen, das über den Hamburger oder in den Milchshake gestreut wird. Wie auf der Zigarettenschachtel gewarnt wird "Rauchen kann tödlich sein", stehen auf dem „MacStatin“ gut lesbar und verständlich die Empfehlungen für die bessere Alternative: gesunde Ernährung, körperliche Aktivität und Rauchverzicht (s. $A b b$.).

H. HolzG ReVE -

\section{- E. A. Ferenczi et al.}

Can a statin neutralize the cardiovascular risk of unhealthy dietary choices? Am. J. Cardiol. 106 (2010) 587-592 für die Umsetzung. In den Fast-Food-Ketten 